\title{
VERIFICAÇÃO DA UTILIZAÇÃO E DA IMPORTÂNCIA DA MEDIÇÃO DA QUALIDADE DA INFORMAÇÃO EM PROCESSOS DE PRODUÇÃO DE PRODUTOS FÍSICOS E DE INFORMAÇÃO
}

\section{CHECKING THE USE AND THE IMPORTANCE OF THE MEASUREMENT OF THE INFORMATION QUALITY IN PRODUCTION PROCESSES OF PHYSICAL AND INFORMATION PRODUCTS}

\author{
Valmir Moro Conque Filho ${ }^{1}$; Fábio Favaretto ${ }^{2}$ \\ ${ }^{1}$ Pontifícia Universidade Católica do Paraná - PUCPR - Curitiba - Brasil \\ valmir.filho@pucpr.br \\ ${ }^{2}$ Pontifícia Universidade Católica do Paraná - PUCPR - Curitiba - Brasil \\ fabio.favaretto@pucpr.br
}

\begin{abstract}
Resumo
A informação tornou-se uma ferramenta extremamente poderosa para o desenvolvimento da estratégia da empresa e conseqüentemente, para se alcançar e se sustentar uma vantagem competitiva e um posicionamento estratégico. Partindo-se dessas necessidades, o surgimento de sistemas de informações, com as suas mais diversas características, formas e finalidades não demorou a acontecer. O presente artigo tem como objetivo principal verificar a utilização e a importância da medição da qualidade da informação (QI) em alguns processos produtivos. Isso será feito através de um instrumento de avaliação da QI aplicado ao gestor elou principal decisor do processo. A pesquisa terá a seguinte estruturação: serão apresentados alguns conceitos sobre qualidade da informação, em seguida, a metodologia utilizada para o desenvolvimento do trabalho e algumas características dos processos produtivos envolvidos. Serão expostos também os resultados gerados e as considerações finais pertinentes. Esperam-se como resultados, a disseminação do conhecimento relativo a QI e principalmente a indicação da importância de se utilizar dados com qualidade em processos de decisão.
\end{abstract}

Palavras-chave: informação, processos produtivos, análise da qualidade da informação.

\section{Introdução}

A informação tornou-se uma ferramenta extremamente poderosa para o desenvolvimento da estratégia da empresa e conseqüentemente, para se alcançar e se sustentar uma vantagem competitiva e um posicionamento estratégico. Como as informações dentro das organizações são geradas de diversas fontes, tanto internas quanto externas e de diferentes formas, através de ofícios, 
memorandos, e-mails, reuniões, a necessidade de se organizar esse tão importante recurso passou a ser imprescindível para a organização.

Partindo-se dessas necessidades, o surgimento de sistemas de informações, com as suas mais diversas características, formas e finalidades não demorou a acontecer. Esses sistemas, além de disponibilizarem as informações de uma maneira mais adequada para os seus usuários, podem ainda, auxiliar os envolvidos em processos decisórios a tomarem uma decisão mais acertada do que os que não possuem tal ferramenta.

As organizações passaram por profundas transformações em decorrência da globalização da economia e dos avanços tecnológicos, não apenas os relacionados à produção, mas especialmente os que dizem respeito à tecnologia da informação, tornando-se importante o papel dos sistemas de informações gerenciais, no sentido de coordenar e aperfeiçoar o fluxo de informações relevantes aos gestores na tomada de decisão (BEUREN e GIBBON, 2001).

Mesmo com todos esses avanços, é difícil garantir que a qualidade dos dados que abastecem esses sistemas seja satisfatória. Estima-se que os custos com problemas relacionados à falta de qualidade nos dados ficam em torno de 8 a 25\% da receita da empresa. Mais do que isso, problemas envolvendo dados sem qualidade podem constituir 40 a $50 \%$ do orçamento destinado à tecnologia da informação da organização (SHANKARANARAYANAN e CAI, 2005).

O presente artigo tem como objetivo principal verificar a utilização e a importância da medição da qualidade da informação (QI) em alguns processos produtivos. Isso será feito através de um instrumento de avaliação da QI aplicado ao gestor e/ou principal decisor do processo.

A pesquisa terá a seguinte estruturação: serão apresentados alguns conceitos sobre qualidade da informação, em seguida, a metodologia utilizada para o desenvolvimento do trabalho e algumas características dos processos produtivos envolvidos. Serão expostos também os resultados gerados e as considerações finais pertinentes. Esperam-se como resultados, a disseminação do conhecimento relativo a QI e principalmente a indicação da importância de se utilizar dados com qualidade em processos de decisão.

\section{Conceitos sobre qualidade da informação}

Considerando que usualmente os termos dados e informações são usados como sinônimos, pois o processamento de dados gera informações e este artigo irá tratar da qualidade da informação, entendendo-se que os dados também devam ter qualidade, o termo qualidade da informação fará referência a qualidade de ambos (FAVARETTO, 2007).

De acordo com Price e Shanks (2005), “a eficácia de uma organização é dependente da QI, que pode ser assegurada somente através de uma política contínua de avaliação e administração". 
Contribuições teóricas relativas à qualidade da informação, se comparada com aplicações práticas da medição da mesma, já existem em maior número na literatura corrente. Muitas das definições de QI derivaram dos já consagrados conceitos existentes sobre qualidade do produto. Strong (1997), afirma que "a definição padrão de qualidade do produto é pronto para uso, conseqüentemente, a informação possui uma qualidade aceitável se ela está pronta para o uso a que foi proposta". Devido a essas derivações, uma analogia entre qualidade do produto e qualidade da informação pode ser elaborada. A manufatura de produtos pode ser vista como um sistema de processamento que age em materiais brutos para produzir produtos físicos. Da mesma forma, a manufatura da informação pode ser vista como um sistema agindo em dados brutos para produzir um produto de informação (WANG; ZIAD e LEE, 2000). A Tabela 1 a seguir, exibe uma analogia entre a manufatura do produto e a manufatura da informação.

Tabela 1 - Analogia entre manufatura do produto e manufatura da informação

\begin{tabular}{lcc}
\hline \multicolumn{1}{c}{ Sistema de manufatura } & $\begin{array}{c}\text { Manufatura do } \\
\text { produto }\end{array}$ & $\begin{array}{c}\text { Manufatura da } \\
\text { informação }\end{array}$ \\
\hline Entrada & Material bruto & Dados brutos \\
Processo & Linha de montagem & Sistema de informação \\
Saída & Produto físico & Produto informação \\
\hline
\end{tabular}

Fonte: Adaptado de Wang, Ziad e Lee (2000)

A abordagem Produto de Informação (PI), de acordo com Shankaranarayan et al (2003), “ganhou considerável aceitação por várias razões: produzir um PI é semelhante a produzir um produto físico; PI, assim como os produtos físicos, podem ser agrupados baseando-se nas características similares e nas entradas de dados comuns, permitindo-se que o grupo possa ser gerenciado como um todo; métodos aprovados para a administração total da qualidade ou, utilizando a nomenclatura em inglês, a Total Quality Management (TQM), tais como qualidade das fontes e melhoria contínua, que têm sido aplicadas com sucesso na produção, podem ser adaptados para o gerenciamento total da qualidade de dados".

Baseando-se ainda na analogia entre dados e produtos manufaturados, uma extensão da TQM para dados foi proposta, a TDQM. De acordo com Wang (1998), o propósito da metodologia TDQM é a "entrega de produtos de informação de alta qualidade para os consumidores. Visa facilitar a implementação em uma organização como um todo de uma política de qualidade de dados formalmente expressa como gerenciamento de alto nível. Fundamental para esta metodologia é a premissa de que as organizações devem tratar informação como um produto que se move através dos sistemas de manufaturas de informações, de forma muito parecida com a de um produto físico". Quatro fases são reconhecidas como necessárias para gerenciar o PI, a definição, a medida, a análise e a melhoria (BERTOLAZZI e SCANNAPIECO, 2001). A TDQM compreende quatro passos. O primeiro passo é definir os requisitos de qualidade e as métricas a serem adotadas. Em segundo, 
deve-se medir a qualidade ao longo do ciclo de vida das informações nos sistemas e processos que as produzem. O terceiro passo é a analise e identificação de potenciais causas dos problemas de qualidade. Por fim, o quarto passo, especifica-se e implementam-se as melhorias nos processos e sistemas para manter a qualidade dos dados e prevenir eventuais vulnerabilidades (WANG, ZIAD e LEE, 2000).

Assim como o produto físico possui indicadores de qualidade, o produto informação possui atributos que cumprem essa finalidade. Esses atributos estão agrupados em categorias. A Tabela 2 apresenta esta definição.

Tabela 2 - Atributos categorizados da qualidade da informação

\begin{tabular}{lc}
\hline \multicolumn{1}{c}{ Categorias } & Atributos \\
\hline $\begin{array}{l}\text { Intrínseca } \\
\text { Acessibilidade } \\
\text { Contextual }\end{array}$ & $\begin{array}{c}\text { Acuracidade, objetividade, credibilidade e reputação } \\
\text { Acessibilidade e segurança no acesso }\end{array}$ \\
$\begin{array}{l}\text { Representação } \\
\text { Relevância, valor agregado, temporalidade e volume apropriado } \\
\text { de informação } \\
\text { Interpretabilidade, facilidade de entendimento, representação } \\
\text { concisa, representação consistente e facilidade de manipulação }\end{array}$ \\
\hline Fonte: Adaptado de Wang, Ziad e Lee (2000)
\end{tabular}

\section{Metodologia}

A pesquisa adotada será descritiva, aplicada em estudos de casos.

Existem três princípios para a coleta dos dados. Esses princípios segundo Yin (2005), “foram muito ignorados no passado e hoje, por fim, são bastante discutidos". Os três princípios são: a utilização de várias fontes de evidência, a criação de um banco de dados para o estudo de caso e a manutenção do encadeamento de evidências.

Os instrumentos de coleta dos dados mais utilizados são, a entrevista, o formulário e o questionário. Na aplicação da entrevista e do formulário, o informante conta com a presença do pesquisador. Já o questionário, sem a presença deste, é preenchido pela pessoa que dá as informações. O tipo de pergunta pode ser fechada, pelo número limitado de opções ou aberta, sem restrições (CERVO e BERVIAN, 2002).

De acordo com Yin (2005), as entrevistas são "uma das mais importantes fontes de informações para um estudo de caso" e de acordo com Cervo e Bervian (2002), a entrevista "é uma conversa orientada para um objetivo definido: recolher, por meio do interrogatório do informante, dados para a pesquisa".

Portanto, o trabalho utilizará a entrevista e a aplicação de um questionário com questões abertas e fechadas, ambos ocorrendo simultaneamente, como instrumentos de coleta dos dados.

Alguns autores como Yin, por exemplo, discutem sobre a utilização de um estudo de caso único ou de casos múltiplos. Apesar de ser somente uma empresa, pode-se considerar que será 
realizado um estudo de casos múltiplos devido ao fato de a coleta dos dados ocorrerem em diferentes processos produtivos, sendo considerado cada processo um caso. De acordo com Yin (2005, p. 75), “quando se tiver escolha, é melhor preferir projetos de casos múltiplos a projetos de caso único. Os projetos de caso único são vulneráveis no mínimo porque se terá apostado todas as fichas num único número. Mais do que isso, os benefícios analíticos de ter dois ou mais casos podem ser substanciais. Com dois casos, se tem a possibilidade de usar replicação direta”. Outros autores, Pozzebon e Freitas (1997), afirmam que “a adoção de múltiplos casos é desejável para a descrição de um fenômeno, a construção ou teste de uma teoria”.

Um questionário a nível gerencial foi elaborado contendo questões abertas e fechadas. As informações relevantes que devem ser coletadas estão dispostas da seguinte maneira:

- Informações relativas à tecnologia da informação;

- Informações relativas à QI.

\section{Processos produtivos}

Os procedimentos adotados para a coleta dos dados serão aplicados somente em uma empresa, mas que possui diversos processos produtivos, podendo-se caracterizar então a utilização de múltiplos estudos de casos. Dentro de cada processo produtivo será entrevistado apenas um decisor, número esse definido, devido ao fato de apesar de existirem mais usuários dentro de cada processo, as decisões são tomadas apenas pelo gestor, os outros integrantes são apenas executores de atividades de rotina. A empresa é uma prestadora de serviços, que atua nas áreas de saúde, educação, comunicação e negócios suplementares.

Devido ao fato de que a empresa possui centenas de processos produtivos, tanto de produtos físicos, quanto de produtos de informação, a necessidade de se delimitar os tipos de processos que serão utilizados para a coleta das informações se torna imprescindível. Para fazerem parte do objeto da pesquisa, os processos, com relação aos seus sistemas de informações deverão ter em comum as seguintes características: produzirem informações que sirvam de apoio a processos decisórios e que essas informações sejam provenientes de diferentes fontes de dados. Após essa delimitação, foi realizado um levantamento do número de processos existentes dentro da empresa com as características supracitadas. Esse levantamento procedeu da seguinte maneira: foi consultada a página na Internet (site) da empresa e verificado, dentro de toda a relação de serviços que a mesma presta, quais serviços são prestados através de processos produtivos e conseqüentemente, os que se encaixam nas condições citadas anteriormente. Foi detectado o número de quatro processos. A Figura 1 a seguir ilustra o ponto de análise dos sistemas de informações dos processos produtivos, onde a pesquisa irá atuar. 
Figura 1 - Ponto de análise

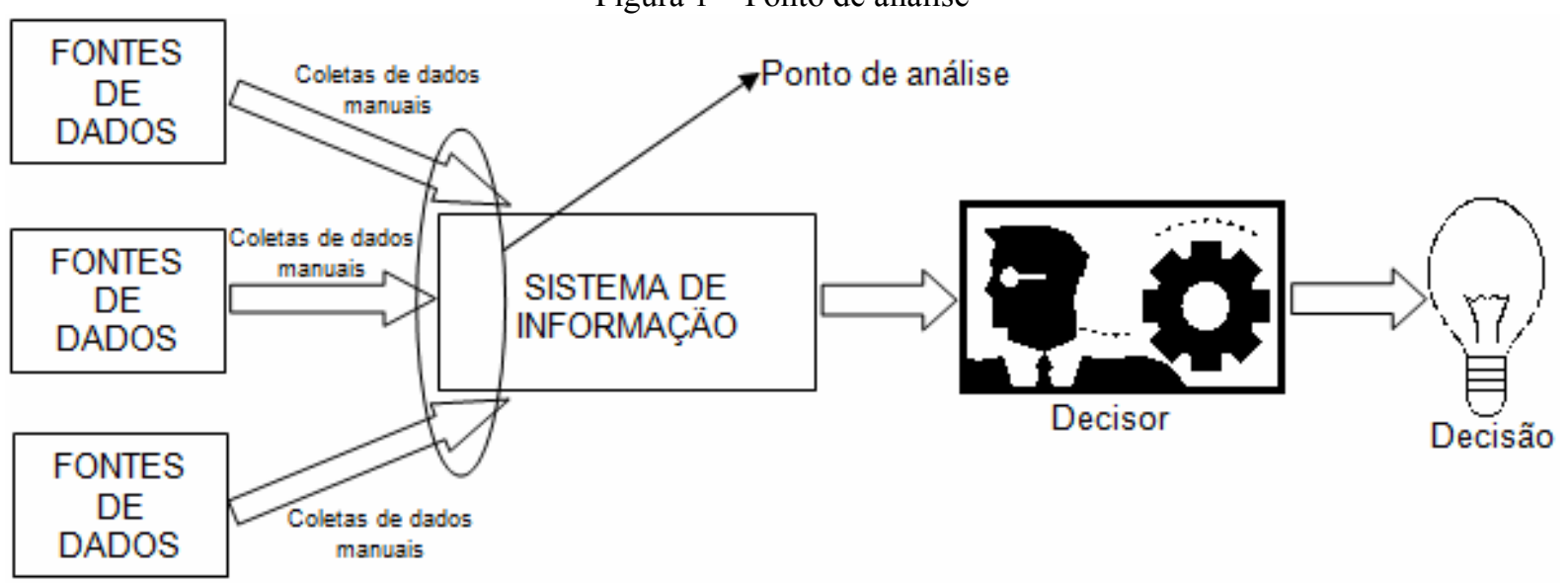

Fonte: Autoria própria (2008)

Esse ponto de análise é a entrada do sistema de informação, ou seja, a alimentação do mesmo através das coletas realizadas de forma manual.

Os quatro processos selecionados possuem outra característica em comum, todos eles produzem e comercializam peixes, sendo somente diferenciados pelas particularidades que serão citadas a seguir. O Processo 1, se caracteriza pela produção e comercialização de peixes de água salgada da Região Sul do Brasil. O Processo 2, produz e comercializa peixes de água doce, de espécies exóticas, mas que já estão adaptadas a Região Sul do Brasil. O Processo 3 também produz e comercializa peixes de água doce, mas se diferencia do Processo 2 pelo fato de trabalhar somente com espécies nativas da mesma região anteriormente identificada. E, para finalizar, o Processo 4, igualmente realiza a produção e a comercialização de peixes de água doce, mas de espécies nativas da Região Nordeste do Brasil.

\section{Apresentação e análise dos resultados}

As perguntas e as respostas serão apresentadas no forma de tabelas (Tabelas 3 a 9) para facilitar a visualização. Para a resposta de algumas questões, será necessária a utilização da Escala Likert apresentada na Figura 2 a seguir.

Figura 2 - Escala Likert

\begin{tabular}{|c|c|c|c|c|}
\hline 1 & 2 & 3 & 4 & 5 \\
\hline EXCELENTE & BOM & NEUTRO & RUIM & PÉSSIMO \\
\hline
\end{tabular}

Fonte: Allen (2007) 
Tabela 3 - Resposta do decisor de cada processo produtivo para a Questão 1 (Avalie o nível de informação, através da Escala Likert, existente nos processos decisórios, justificando a escolha) do questionário

\begin{tabular}{|c|c|}
\hline Processo & Resposta \\
\hline Processo 1 & $\begin{array}{l}\text { Valor da escala: 3. Justificativa: ainda falta a conscientização da } \\
\text { importância de se registrar informações por parte da equipe } \\
\text { envolvida }\end{array}$ \\
\hline Processo 2 & $\begin{array}{c}\text { Valor da escala: 4. Justificativa: a coleta de dados ainda é } \\
\text { realizada manualmente }\end{array}$ \\
\hline Processo 3 & $\begin{array}{c}\text { Valor da escala: 2. Justificativa: o nível de informatização está } \\
\text { dentro das necessidades do processo }\end{array}$ \\
\hline Processo 4 & $\begin{array}{l}\text { Valor da escala: 2. Justificativa: praticamente todos os recursos } \\
\text { disponíveis estão sendo utilizados }\end{array}$ \\
\hline
\end{tabular}

Analisando a Questão 1, dois processos produtivos avaliaram o nível de informatização, como bom dentro da escala Likert, justificando que a tecnologia existente hoje atende as necessidades dos mesmos. As outras duas avaliações que receberam valores da escala entre neutro e ruim, são justificadas pelos fatos de que em um dos processos, muitas das informações úteis não são coletadas e no outro, a coleta é realizada manualmente.

Tabela 4 - Resposta do decisor de cada processo produtivo para a Questão 2 (As informações que abastecem os sistemas de informações são coletadas de que(ais) maneira(s)?) do questionário

\begin{tabular}{ll}
\hline \multicolumn{1}{c}{ Processo } & Resposta \\
\hline Processo 1 & Manualmente \\
Processo 2 & Manualmente \\
Processo 3 & Manualmente \\
Processo 4 & Manualmente \\
\hline
\end{tabular}

Fonte: Entrevista realizada com o decisor de cada processo (2008)

Analisando a Questão 2, verifica-se que em todos os processos produtivos, os sistemas de informações utilizados nos processos de decisão são abastecidos com dados coletados de forma manual.

Tabela 5 - Resposta do decisor de cada processo produtivo para a Questão 3 (Dentre os sistemas: Sistemas de apoio a decisão (SADs), especialistas, executivos, gerenciais e transacionais, como são classificados os utilizados nos processos decisórios?) do questionário

\begin{tabular}{lc}
\hline \multicolumn{1}{c}{ Processo } & Resposta \\
\hline Processo 1 & SADs e transacionais \\
Processo 2 & SADs, gerenciais e transacionais \\
Processo 3 & Gerenciais \\
Processo 4 & SADs e gerenciais \\
\hline Fonte: Entrevista realizada com o decisor de cada processo $(2008)$
\end{tabular}

Analisando a Questão 3, verifica-se que, dentre os cinco tipos de sistemas apresentados na questão, apenas três são citados nas respostas dos decisores. 
Tabela 6 - Resposta do decisor de cada processo produtivo para a Questão 4 (Avalie, através da Escala Likert, o nível de confiabilidade existente nas(s) fonte(s) de coleta(s) de dados e justifique) do questionário

\begin{tabular}{|c|c|}
\hline Processo & Resposta \\
\hline Processo 1 & $\begin{array}{c}\text { Valor da escala: } 4 \text {. Justificativa: os Sis são confiáveis, mas como } \\
\text { as coletas são manuais não se pode confiar plenamente. }\end{array}$ \\
\hline Processo 2 & $\begin{array}{l}\text { Valor da escala: 4. Justificativa: a maioria das coletas são } \\
\text { manuais, gerando desconfianças. }\end{array}$ \\
\hline Processo 3 & $\begin{array}{l}\text { Valor da escala: 2. Justificativa: a seleção da fonte é definida pelo } \\
\text { decisor, aumentando a confiabilidade das mesmas. }\end{array}$ \\
\hline Processo 4 & $\begin{array}{c}\text { Valor da escala: 2. Justificativa: apesar da seleção ser feita pelo } \\
\text { decisor, não se pode confiar totalmente. }\end{array}$ \\
\hline
\end{tabular}

Analisando a Questão 4, verifica-se que dois processos avaliaram o nível de confiabilidade, de acordo com a escala Likert como ruim, devido ao fato das coletas de dados ocorrerem manualmente. Nos outros dois processos, a seleção das fontes de coletas de dados é feita pelo decisor e mesmo assim ficaram avaliadas como bom, verificando-se com isso, que não se pode confiar totalmente nas fontes.

Tabela 7 - Resposta do decisor de cada processo produtivo para a Questão 5 (Dentre os atributos citados na Tabela 2 , ordene, em ordem crescente, os cinco mais importantes) do questionário

\begin{tabular}{cc}
\hline Processo & Resposta \\
\hline Processo 1 & Relevância, acuracidade, credibilidade, interpretabilidade, \\
acessibilidade & \\
Processo 2 & Credibilidade, acessibilidade, objetividade, temporalidade, volume \\
Processo 3 & Credibilidade, objetividade, acessibilidade, segurança, relevância \\
Processo 4 & Volume, acessibilidade, objetividade, facilidade de manipulação, \\
& temporalidade \\
\hline
\end{tabular}

Fonte: Entrevista realizada com o decisor de cada processo (2008)

Analisando a Questão 5, verifica-se que apenas dez dos quinze atributos apresentados na Tabela 2 foram citados pelos decisores na resposta da Questão 5. A Tabela 8 a seguir, apresenta o número de vezes que cada atributo foi citado.

Tabela 8 - Número de vezes que cada atributo foi citado na resposta da Questão 5

\begin{tabular}{lc}
\hline \multicolumn{1}{c}{ Atributo } & Número de vezes \\
\hline Acessibilidade & 4 \\
Credibilidade & 3 \\
Objetividade & 3 \\
Relevância & 2 \\
Temporalidade & 2 \\
Volume & 2 \\
Acuracidade & 1 \\
Facilidade de manipulação & 1 \\
Interpretabilidade & 1 \\
Segurança & 1 \\
\hline Fonte: Entrevista realizada como
\end{tabular}

Fonte: Entrevista realizada com o decisor de cada processo (2008) 
Tabela 8 - Resposta do decisor de cada processo produtivo para a Questão 6 do questionário

\begin{tabular}{cc}
\hline & Questão 6: Dê sua opinião com relação à medição da QI \\
\hline Processo 1 & $\begin{array}{c}\text { A medição é de extrema importância, pois influencia diretamente } \\
\text { na velocidade e na probabilidade de se tomar uma decisão mais } \\
\text { correta }\end{array}$ \\
Processo 2 & $\begin{array}{c}\text { A medição da qualidade da informação é uma metodologia } \\
\text { imprescindível para qualquer processo decisório, pois ela dá um } \\
\text { forte embasamento ao decisor, tornando o árduo processo de se } \\
\text { tomar decisões uma tarefa fácil e com a garantia de se ter tomado a } \\
\text { decisão mais correta }\end{array}$ \\
Processo 3 & $\begin{array}{c}\text { É importante medir a qualidade da informação, pois os dados serão } \\
\text { utilizados para se tomar decisões fundamentais no processo } \\
\text { produtivo }\end{array}$ \\
Processo 4 & $\begin{array}{c}\text { A medição da qualidade da informação é muito importante, mas é } \\
\text { preciso cuidado para que não se torne um trabalho que envolva } \\
\text { muito esforço, prejudicando o desenvolvimento do processo } \\
\text { produtivo }\end{array}$ \\
\hline Fonte: Entrevista realizada com o decisor de cada processo produtivo
\end{tabular}

Analisando a Questão 6, verifica-se que todos os decisores afirmaram que a medição da qualidade da informação é fundamental para os processos decisórios, destacando-se ainda que ao se utilizar dados com qualidade, a decisão pode vir a ser a mais correta. Uma contribuição que merece destaque é a do Processo 4, onde o decisor comenta sobre o esforço gasto para se medir a qualidade da informação, pois de acordo com o mesmo, é preciso tomar cuidado para que não se torne um trabalho que exija tanta dedicação que acabe prejudicando as atividades do processo produtivo propriamente dito.

\section{Considerações finais}

Através das respostas dos decisores para as questões do questionário, verifica-se que as informações utilizadas nos processos decisórios em que os mesmos atuam, além de serem coletadas manualmente, sendo portanto, mais suscetíveis a erros humanos, não possuem nenhum método de medição da QI, concluindo-se com isso que, processos que não adotam práticas estruturadas de gestão da informação estão mais expostas a riscos e falhas do que as que possuem tais ferramentas.

É possível também concluir que, apesar da não utilização, por parte do processo, de metodologias para a medição da QI, de acordo com os entrevistados, a medição da QI é de extrema importância para os processos decisórios.

Como conclusão final, a implementação da medição da qualidade da informação, ou seja, se as informações utilizadas possuírem, no mínimo, as características, ou os atributos selecionados pelos gestores, as mesmas já se encontram num grau de confiabilidade necessário para a utilização nos processos de decisão. 


\begin{abstract}
The information became an extremely tool for the development of the strategy of the enterprise and consequently, to reach and were supported a competitive advantage and a strategic positioning. Because of these necessities, the appearance of information systems, with more several characteristics, forms and finalities did not delay to happening. The present article has like principal objective checks the use and the importance of the measurement of the information quality (IQ) in some productive processes. That will be done through an instrument of evaluation of the IQ applied to the manager and/or principal decisor from the process. The research will have the next structuring: there will be presented some concepts of information quality, next, the methodology used for the development of the work and some characteristics of the productive process used. There will be exposed also the produced results and the final relevant considerations. They are waited as results, the dissemination of the relative knowledge to IQ and principally the indication of the importance of being used information with quality in decision processes.
\end{abstract}

Key-words: information, productive processes, analysis of the information quality.

\title{
Referências
}

BERTOLAZZI, P.; SCANNAPIECO, M. Introducing Data Quality in a Cooperative Context. Proceedings of the $6^{\text {th }}$ International conference on Information Quality Management Science. Vol. 44, n. 4, p. 462-484, 1998

BEUREN, I. M.; GIBBON, A. R. de O. Considerações Acerca do Sistema de Informação Gerencial Como Suporte ao Controle de Gestão: uma Abordagem da Gestão do Conhecimento. Anais do XXI Encontro Nacional de Engenharia de Produção, Salvador, Bahia, Brasil, 2001, p. 1-8.

BRYMAN, A. Research Methods and Organization Studies. London: Routledge, 1989.

CERVO, A. L.; BERVIAN, P. A. Metodologia Científica. 5. ed. São Paulo: Pearson Prentice Hall, 2002.

FAVARETTO, F. Experimento Para Análise da Implantação da Medição da Qualidade da Informação. Produção. Vol. 17, n. 1, p. 151-161, 2007.

FAVARETTO, F.; MATTIODA, R. A. Medição da Qualidade da Informação: um Experimento na Pesquisa em Bases de Dados Científicas. Anais do XXV ENEGEP, Porto Alegre, Rio Grande do Sul, Brasil, 2005, p. 4572-4578.

MARTINS, R. A.; SALERNO, M. S. Sistemas de Medição de Desempenho: um Modelo para Estruturação do Uso. Escola Politécnica da Universidade de São Paulo, São Paulo, 1999.

MATTIODA, R. A.; FAVARETTO, F. Qualidade de Informação em Empresas que Utilizam Data Warehouse na Perspectiva do Consumidor de Informação. Pontifícia Universidade Católica do Paraná, Curitiba, 2006.

POZZEBON, M.; FREITAS, H. M. R. Pela Aplicabilidade - Com Maior Rigor Científico - dos Estudos de Caso em Sistemas de Informação. $21^{\circ}$ Encontro da Associação Nacional de Pós-Graduação e Pesquisa em Administração ANPAD, Rio das Pedras, 1997, p. 1-15.

PRICE, R.; SHANKS, G. Empirical Refinement of a Semiotic Information Quality Framework. Proceedings of $38^{\text {th }}$ Hawaii International Conference on System Sciences, Hawaii, p. 1-10, 2005.

SHANKARANARAYANAN, G.; CAI, Y. Supporting Data Quality Management in Decision-making. Decision Support Systems. 2005.

SHANKARANARAYANAN, G., et al. Managing Data Quality in Dynamic Decision Environments: an Information Product Approach. Journal of Data Management. P. 1-24, 2003. 
STRONG, D. M. IT Process Designs for Improving Information Quality and Reducing Exception Handling: A Simulation Experiment. Information \& Management. Vol. 31, p. 251-263, 1997.

crossef

WANG, R. Y. A Product Perspective on Total Data Quality Management. Comunications of the ACM. Vol. 41, n. 2, p. $58-65,1998$.

cross ${ }^{\text {ref }}$

WANG, R. Y; ZIAD, M.; LEE, Y. W. Data quality. Massachusetts: Kluwer Academic Publishers, 2000.

YIN, R. K. Estudo de Caso: Planejamento e Método. 3. ed. Porto Alegre: Bookman, 2005.

\section{Dados dos autores:}

Nome completo: Valmir Moro Conque Filho

Filiação institucional: Pontifícia Universidade Católica do Paraná - PUCPR

Departamento: Programa de Pós-Graduação em Engenharia de Produção em Sistemas - PPGEPS

Função ou cargo ocupado: Ex-Aluno Mestrado

Endereço completo para correspondência (bairro, cidade, estado, país e CEP): Travessa Tereza

Francisca Dissenha Conque, 43 - Silveira da Motta - 83030-495 - São José dos Pinhais-PR

Telefones para contato: (41) 3282-3394 / (41) 9125-6215

e-mail:vmc_filho@hotmail.com

Nome completo: Fábio Favaretto

Filiação institucional: Pontifícia Universidade Católica do Paraná - PUCPR

Departamento: Programa de Pós-Graduação em Engenharia de Produção em Sistemas - PPGEPS

Função ou cargo ocupado: Professor Adjunto

Endereço completo para correspondência (bairro, cidade, estado, país e CEP): Rua Imaculada Conceição, 1155 - Prado Velho - 80215-901 - Curitiba-PR

Telefones para contato: (41) 3271-2579 / (41) 3271-1333

e-mail:fabio.favaretto@pucpr.br

Recebido para publicação em: 26/03/2009

Aceito para publicação em: 04/06/2009 